\title{
Result of Applying Laparoscopic Total Gastrectomy with D2 Lymph Node Dissection with Left-Site Surgeon and Final Resection and Closure of the Duodenal Stump in Gastric Cancer Treatment
}

\author{
Van-Huong Nguyen ${ }^{1}$, Van-Chien Dinh ${ }^{1}$, Van-Quyet $\mathrm{Ha}^{2}$, Van-Duyet Pham², Van-Thuong Pham², \\ Quoc-Ai Dang ${ }^{3^{*}}$
}

'Nghe An Friendship General Hospital

2Haiphong University of Medicine and Pharmacy

${ }^{3}$ Department of Surgery, Ha Noi Medical University Hospital

\section{ABSTRACT}

Objective: To evaluate the outcomes, safety and effectiveness of laparoscopic total gastrectomy with D2 lymph node dissection with left-site surgeon and resection - closure of the duodenal stump in final phase after making esophageal anastomosis in gastric cancer treatment.

Method: Prospective, descriptive study on patients undergoing laparoscopic total gastrectomy and D2 lymphadenectomy with left-site surgeon and resection - closure of the duodenal stump in final phase after making esophageal anastomosis in gastric cancer treatment from 06/2016 03/2020.

Results: There were 70 patients in the study. The mean age was 59.9 years old, with a male/female ratio of $4 / 1$. ASA 1 and 2 accounted for $97.2 \%$ of patients. The average lesion size was $3.4 \mathrm{~cm}$. In total, $78.6 \%$ of patients had adenocarcinoma; $15.7 \%$ cases had tumour sites found in the upper third of the stomach, and $75.7 \%$ were found in middle third of stomach; $4.3 \%$ of cancers were at stage IA, $11.4 \%$ at IB, $32.9 \%$ at IIA, $30 \%$ at stage IIB, $7.1 \%$ at IIIA, $11.4 \%$ IIIB and $2.9 \%$ IIIC; $100 \%$ patients received laparoscopic total gastrectomy and D2 lymph node dissection with left-site surgeon and posterior duodenectomy; $4.2 \%$ had minor complications during surgery, and $2.8 \%$ developed complications after surgery; no deaths occurred during and after surgery. The average number of retrieved lymph nodes was 21.5 nodes, the average number of metastatic lymph nodes was 2.6, and the lymph node station numbers 1, 2, 3, 4 and 7 had high rates of metastases. The mean surgical time was 206.8 minutes, and the mean hospital stay was 8.8 days. The mean overall survival was estimated as 43.8 months.

Conclusion: Laparoscopic total gastrectomy with D2 lymphadenectomy with left-site surgeon and final resection and closure of the duodenal stump demonstrated its safety and effectiveness in gastric adenocarcinoma treatment with less pain for patients, earlier movement recovery and lower hospitalization time.

Key words: Laparoscopic total gastrectomy with D2 lymph node dissection for gastric cancer

\section{INTRODUCTION}

Gastric cancer is one of the most prevalent causes of cancer-related death in

\author{
*Corresponding author: \\ Quoc-Ai Dang, MD \\ Department of Surgery \\ Ha Noi Medical University Hospital \\ E-mail: drdangquocai@gmail.com
}


many countries around the world, such as in Japan, Korea, China, and Vietnam. Currently, surgery is still the most effective treatment (1). Azagra was the first person in the world to perform laparoscopic surgery for total gastrectomy in 1993 (2). In Vietnam, laparoscopic gastrectomy (LG) was first conducted in 2004. Up to now, this method has been widely applied in gastric cancer treatment and has confirmed the value of cancer dismantlement. The potential advantages of laparoscopic gastrectomy include minimal invasion, decreased pain, faster recovery, less blood loss, and lower rate of postoperative complications, which have improved the quality of life and extended the survival time for patients (3-5).

However, laparoscopic total gastrectomy with lymph node dissection is still associated with some problems related to surgical technique, applicability and extent of lymphadenectomy as well as postoperative survival time, which are different in different countries worldwide.

To have a separate and clear view of total laparoscopic total gastrectomy with lymph node dissection, we performed this study, with the aim of evaluating results of laparoscopic total gastrectomy and D2 lymph node dissection with the left-site surgeon and final resection and closure of the duodenal stump in gastric cancer treatment.

\section{METHOD}

\section{Study subjects}

The study subjects included 70 patients who were assigned to laparoscopic total gastrectomy and D2 lymph node dissection with left-site surgeon and resection - closure of the duodenal stump in final phase after making esophageal anastomosis in gastric cancer treatment at Nghe An General Hospital, Vietnam from June 2016 to March 2019.

\section{Study design}

Prospective and descriptive study.

\section{Patient selection}

Patients with stage IA - IIIC gastric cancer, lesions on $2 \beta$ of the stomach or lesions with margins $<6 \mathrm{~cm}$ from the cardia.

\section{Study criteria}

Patient characteristics: Age, gender, ASA, pain symptoms, weight loss, lesion site, pathology, stage of disease, gastrectomy method, and anastomosis.

Surgical outcome: Rate of conversion to open surgery, extent of lymphadenectomy and lymph node metastases, intraoperative complications, and degree of postoperative pain

Early results: Time to first flatus, time of gastric catheter removal, time of feeding after surgery, and postoperative hospital stay.

Further results: Overall survival and disease-free survival.

Surgical Procedure: We performed the steps according to the surgical procedure as follows:

The patient was placed in supine position under general anaesthesia; the surgeon stood on the left side of the patient.

A total of five operating trocars were used: one initial 10-mm trocar was inserted for laparoscopy below the umbilicus, one 5-mm trocar was introduced at the right flank, one 12-mm trocar was insert at left flank and two 5-mm trocars were introduced at both sides of the upper quadrant.

Step 1: Assess the lesion and possibility for gastrectomy.

Step 2: Dissect the greater omentum along the border of the transverse colon with ultrasonic coagulating shears, completely remove the spinal conjunctiva, group 4d, 4sb, 4sa, 11d, 10 and 2, and continue the dissection towards the left side of the patient until the oesophageal cardia is visualized.

Step 3: Dissect lymph node station Nos. 6. The bifurcation of the gastroduodenal artery and right gastroepiploic artery are divided, and the right gastroepiploic vessel is clamped at its origin and cut.

Step 4: Dissect lymph node station Nos. 3, 5, 8a, and 12a. Ligate the right artery, which is near the liver artery.

Step 5: Dissect lymph node station Nos. 7, 9, and 11p. Perform ligation of the left vein with clips near the base of the artery and ligation of root artery close to the celiac artery.

Step 6: The remaining small omentums are removed, and paracardial lymph node No. 1 is dissected. Expose and move the oesophageal cardia.

Step 7: Functional end-to-end oesophagojejunostomy anastomosis is performed using linear staplers, and the entry hole is hand-sewn closed.

Step 8: The pylorus is identified and the duodenum is transected $1-1.5 \mathrm{~cm}$ distally by linear stapler to create a secure sealing of the duodenal stump, and the stomach and omentum are placed in the lab sample bag. 
Step 9: The abdomen is cleaned. A drain is inserted under the liver and splenic cavity.

Step 10: The 12-mm trocar incision at left flank is enlarged $2 \mathrm{~cm}$ to bring out the stomach and omentum, and all of the trocar holes are closed.

\section{Statistical analysis}

All statistical analyses were performed with SPSS version 22.0.

\section{RESULTS}

From 6/2016 - 3/2020, seventy patients underwent laparoscopic total gastrectomy and D2 lymph node dissection with left-site surgeon and final duodenectomy in treatment for gastric cancer.

Table 1 shows the clinical characteristics and pathologic features of the patients. The mean age was $59.9 \pm 9.1$ years, while the lowest was 29 years and the highest was 83 years; the most common age group was 50-70, accounting for $74.2 \%$. The male/female ratio was 4/1. Patients who had American Society of Anesthesiologists (ASA) physical status classifications of 2 accounted for the highest percentage of the study sample (68.6\%), followed by patients who had ASA class $1(28.6 \%)$ and 3 (2.9\%). A total of $97.1 \%$ of patients had abdominal pain, and $87.1 \%$ experienced weight loss before hospital admission. The majority of neoplasms were located in the middle third of the stomach (75.7\%), while $15.7 \%$ of cases had neoplasms found in the upper third of the stomach, and $8.6 \%$ presented ulcerating tumour invasion into the entire stomach. The mean neoplasm size on endoscopy was estimated as $3.4 \pm 1.4 \mathrm{~cm}$. Related to pathology type, $78.6 \%$ patients had ductal adenocarcinoma, while $64.3 \%$ displayed poorly undifferentiated adenocarcinoma.

All gastric cancer patients received laparoscopic total gastrectomy and D2 lymph node dissection with left-site surgeon and resection - closure of the duodenal stump in final phase after making esophageal anastomosis. Minor complications occurred in $4.2 \%$ of cases ( $3 / 70$ patients), and they were treated during laparoscopic surgery. The rate of postoperative morbidity was $2.8 \%$ (2/70 patients), which included residual abscess and gastrointestinal fistula, both of which required internal medicine treatment. There was no perioperative mortality (table 2).

The lymph node stations with high metastases were Nos. 1, 2, 3, 4 and 7. There were no lymph node metastases found in Nos. 10, 12a. The total number of dissected lymph nodes was 1506 nodes, while the mean number was $21.5 \pm 8.3(11-45)$ nodes. The total number of metastatic lymph node was 181 nodes, and the average number of metastatic lymph nodes was 2.6 \pm 3.5 (0-13) nodes (table 3).

Lymph node metastases occurred in $52.9 \%$ of patients, which were mostly found at stage N1, N2, and N3. One hundred percent of upper and lower resection margins had no cancer cells. The TNM stages were distributed as follows: IA (4.3\%); IIB (11.4\%); IIA (32.9\%); IIIB (30.0\%); IIIA (7.1\%); IIIB (11.4\%); and IIIC (2.9\%) (table 4).

The operative results are presented in table 5 . The

Table 1 - Patient characteristics

\begin{tabular}{|c|c|c|}
\hline Mean age (Range) & & $59.9 \pm 9.1$ years $(29-83)$ \\
\hline Male/Female & & $4 / 1$ \\
\hline ASA & $\begin{array}{l}\text { ASA } 1 \\
\text { ASA } 2 \\
\text { ASA } 3\end{array}$ & $\begin{array}{c}20(28.6 \%) \\
48(68.6 \%) \\
2(2.9 \%)\end{array}$ \\
\hline Epigastric abdominal pain & & $68(97.1 \%)$ \\
\hline Weight loss & & $61(87.1 \%)$ \\
\hline Tumour location & $\begin{array}{l}\text { Upper third of the stomach } \\
\text { Middle third of the stomach } \\
\text { Ulcerating tumour invasion }\end{array}$ & $\begin{array}{c}11(15.7 \%) \\
53(75.7 \%) \\
6(8.6 \%)\end{array}$ \\
\hline Lesion size & & $3.4 \pm 1.4(1-7) \mathrm{cm}$ \\
\hline Pathology & $\begin{array}{l}\text { Ductal adenocarcinoma } \\
\text { Mucinous adenocarcinoma } \\
\text { Signet ring cell carcinoma }\end{array}$ & $\begin{array}{c}52(74.3 \%) \\
3(4.3 \%) \\
15(21.4 \%)\end{array}$ \\
\hline Degree of differentiation & $\begin{array}{l}\text { High } \\
\text { Moderate } \\
\text { Poor }\end{array}$ & $\begin{array}{c}2(2.9 \%) \\
23(32.9 \%) \\
45(64.3 \%) \\
\end{array}$ \\
\hline
\end{tabular}


Table 2 - Intraoperative and postoperative complications

\begin{tabular}{lcc}
\hline Complications & N & $\%$ \\
\hline Splenic injury during surgery & 1 & 1.4 \\
\hline Liver damage during surgery & 1 & 1.4 \\
\hline Small intestine muscle tear & 1 & 1.4 \\
\hline Pneumonia & 1 & 1.4 \\
\hdashline Residual abscess after surgery & 1 & 1.4 \\
\hline Gastrointestinal fistula after surgery & 0 & 0.0 \\
\hline Other & 0 & 0.0 \\
\hline
\end{tabular}

operation was completed successfully in all cases without conversions to open surgery. The mean operation time was estimated as $206.8 \pm 27.2$ minutes, and the highest operative time was 300 minutes. The cases with longer surgical time were the ones who received operations in the early stage of applying laparoscopic total gastrectomy with $\mathrm{D} 2$ lymphadenectomy in gastric cancer treatment; the operative time then decreased significantly in later surgical cases in the study. With regard to postoperative pain, we found that $60 \%$ of cases had minor pain, and $38.6 \%$ had moderate pain; no cases had major pain. According to the KaplanMeier method, the 1- and 3-year disease-free survival rates were $97.7 \%$ and $83.8 \%$, respectively.

The 1- and 3-year overall survival rates were $97.7 \%$ and $83.8 \%$, respectively. The mean overall survival was calculated as $43.8 \pm 1.5$ months, fluctuating from 40.9 months to 46.7 months (figs. $1 \& 2$ ).

\section{DISCUSSION}

The mean age in the study was $59.9 \pm 9.1$ years; the lowest age was 29 years, and the highest was 83 years. The most popular age group was 50 to 70 years old, amounting to $74.2 \%$ of cases. The male/female ratio was $4 / 1$. The age of gastric cancer patients in our study was consistent with the findings of previous studies from countries that show common ages of having gastric cancer: 62.7-64.8 years old in Japan (6), 63.6-73 years old in Europe and America (7), and 54.6-57.6 years old in South Korea and China. We assigned total gastrectomy to cases with gastric cancer located in the cardia and body of the stomach, ulcerating gastric adenocarcinoma and gastric cancer cases with lesions $<6 \mathrm{~cm}$ distant from the gastric cardia to ensure excision of tumour organization. This distance was revealed to be $6.9 \pm 0.8 \mathrm{~cm}$ in research by Cristiano et al (7), which reported a shortest distance of $6 \mathrm{~cm}$ and a longest distance of $8 \mathrm{~cm}$. Matsuo Ryota et al (2016) suggested that gastric resection margins must be at least $5 \mathrm{~cm}$ away from the tumour. For ulcerating gastric adeno-
Table 3 - Rates of lymph node metastasis

\begin{tabular}{l|cc}
\hline Lymph nodes station number & N & $\%$ \\
\hline 1 & 29 & 41.4 \\
\hline 2 & 13 & 18.6 \\
\hline 3 & 21 & 30.0 \\
\hline 4 & 14 & 20.0 \\
\hline 5 & 4 & 5.7 \\
\hline 6 & 6 & 8.6 \\
\hline 7 & 11 & 15.7 \\
\hline $8 \mathrm{a}$ & 5 & 7.1 \\
\hline 9 & 3 & 4.3 \\
\hline 10 & 0 & 0.0 \\
\hline 11 & 2 & 2.9 \\
\hline $12 \mathrm{a}$ & & 0 \\
\hline
\end{tabular}

Table 4 - Extent of lymph node metastasis

\begin{tabular}{lcc}
\hline $\begin{array}{l}\text { Extent of lymph node metastases } \\
\text { and disease stage according to TNM }\end{array}$ & N & $\%$ \\
\hline N0 (no metastasis) & 33 & 47.1 \\
\hline N1 (metastasis in 1-2 nodes) & 13 & 18.6 \\
\hline N2 (metastasis in 3-6 nodes) & 14 & 20.0 \\
\hline N3a (metastasis in 7-15 nodes) & 10 & 14.3 \\
\hline N3b (metastasis in >15 nodes) & 0 & 0.0 \\
\hline M1 (distant metastasis) & 0 & 0.0 \\
\hline IA (T1N0) & 3 & 4.3 \\
\hline IB (T1N1, T2N0) & 8 & 11.4 \\
\hline IIA (T1N2, T2N1, T3N0) & 23 & 32.9 \\
\hline IIB (T1N3, T2N2, T3N1, T4aN0) & 21 & 30.0 \\
\hline IIIA (T2N3, T3N2, T4aN1) & 5 & 7.1 \\
\hline IIIB (T3N3, T42N2, T4bN0, T4bN1) & 8 & 11.4 \\
\hline IIIC (T4aN3, T4bN2, T4bN3) & 2 & 2.9 \\
IV (Any T, Any N, M1) & 0 & 0.0 \\
\hline
\end{tabular}

Table 5 - Postoperative results

\begin{tabular}{lccc}
\hline Time & Mean & Lowest & Highest \\
\hline $\begin{array}{l}\text { Surgical time } \\
\text { (minutes) }\end{array}$ & $206.8 \pm 27.2$ & 160 & 300 \\
\hline $\begin{array}{l}\text { Gastric catheter removal } \\
\text { after surgery (hours) }\end{array}$ & $50.1 \pm 34.6$ & 0 & 148 \\
\hline Time to first flatus & $55.3 \pm 20.9$ & 24 & 120 \\
\hline Drainage tube removal (days) & $4.05 \pm 1.3$ & 2 & 7 \\
\hline Postoperative hospital stay & $8.8 \pm 2.4$ & 6 & 15 \\
\hline Disease-free survival & $43.8 \pm 1.5$ & 40.9 & 46.7 \\
\hline
\end{tabular}

carcinoma, the gastric resection portion is under or over the pylorus to the duodenum, and the upper part must at least $6-8 \mathrm{~cm}$ from the tumour margin. For tumours in the body and upper third of the stomach, 


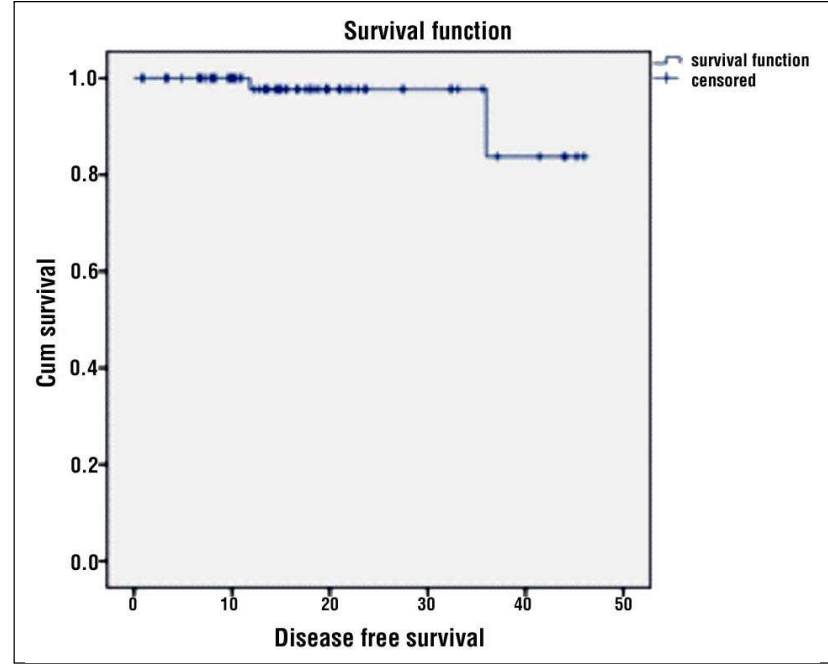

Figure 1 - Disease-free survival

total gastric resection was recommended. The distance from the upper margin of the lesion to the upper resection margin plays an important role in laparoscopic gastrectomy. To prevent recurrence at the anastomosis, the latest recommendation from the Japan and American Association of Gastric Cancer suggested that the incision is at least $5 \mathrm{~cm}$. We performed an oesophageal transection 2-3 cm away from the cardia of the stomach and duodenal resection below the pylorus at a distance of $1-1.5 \mathrm{~cm}$. The study results showed that $15.7 \%$ of patients had lesions located in upper third of the stomach, $75.7 \%$ in the middle third of the stomach and $8.6 \%$ of cases with infected ulcers. The distance from the upper lesion margin to the upper average section was $5.09 \pm 0.9(3-7) \mathrm{cm}$, and $100 \%$ of the upper and lower sections in the study had no cancer cells. The cases with lesions in the fundus are because the cancerous lesion margin is less than $6 \mathrm{~cm}$ distant from the cardia, so to ensure the removal of cancer cell organization, we recommend total gastrectomy.

In our study, $100 \%$ of patients experienced laparoscopic total gastrectomy with D2 lymph node dissection with left-site surgeon and resection - closure of the duodenal stump in final phase after making esophageal anastomosis by linear stapler; no cases were converted to open surgery due to complications. We found that the surgeon standing on the left and excising the posterior duodenum made it favourable and easier to anatomize the vascular bundles around the stomach and dissect lymph nodes because the majority of the mesenteric root is on the right, and the oesophageal cardia area is not obscured by the liver. The aim of duodenal resection after gastrectomy and lymph node dissection is to make 2 fixed points on both

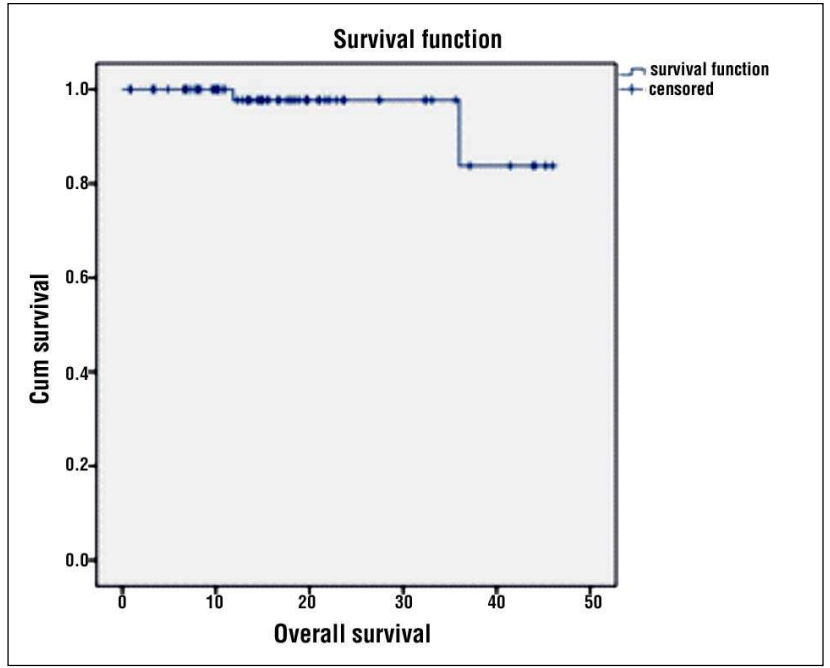

Figure 2 - Overall survival

ends of the stomach, from which we raise the stomach for dissection; the lymphadenectomy also becomes more convenient thanks to the fixed point of the duodenum pylorus, so making an oesophago-jejunal anastomosis without resection of the oesophagus and front jejunum will become more favourable and reduce manipulation, which shortens the operation time and decreases the number of linear staplers (2 staplers), therefore reducing surgical cost.

Oesophagojejunostomy was performed according to the functional end-to-end method with linear staplers (2-3 Echelon Stapler 60B) (9-12). We realize that laparoscopic suture has many advantages compared to anastomosis in laparoscopic-assisted surgery or open surgery, such as easy manipulation for the surgeon, small incisions, decreased postoperative pain, better aesthetics and no cases of gastrointestinal fistulae or anastomosis stenosis after surgery.

We applied the method of D2 lymph node dissection in total gastrectomy for gastric cancer treatment according to the Japanese Gastric Cancer Association (2011) (13-15). The total number of retrieved lymph nodes was 1506, and the average number of dissected lymph nodes was $21.5 \pm 8.3(11-45)$. The total number of metastatic lymph nodes was 181 nodes, and the average number of metastatic lymph nodes was $2.6 \pm$ $3.5(0$ - 13). The lymph node stations with high metastases included No. 1 (41.4\%), No. 2 (18.6\%), No. 3 $(30 \%)$, No. $4(20 \%)$ and No. 7 (15.7\%). No lymph node metastases were observed in Nos. 10, 12 and. The numbers of harvested lymph nodes from previous studies in Western countries are lower, ranging from 18-30.3 nodes (7), (16). In accordance with UICC, AJCC and JRSGC classifications of gastric cancer (13-15), our 
study results found that $47.1 \%$ of cases were at stage No, $18.6 \%$ at stage N1, 20\% at stage N2 and $14.3 \%$ at stage N3; no cases were at stage N3b. D2 lymph node dissection in laparoscopic gastrectomy with the left-site surgeon and resection - closure of the duodenal stump in final phase after making esophageal anastomosis in gastric cancer treatment is completely feasible but must be performed in a fully equipped health facility and have a team of surgeons trained in laparoscopic surgery, with regularly updated in-depth knowledge. Applying TNM disease stage classification following the Union Internationale Contre le cancer (UICC) $7^{\text {th }}$ edition (2009), American Joint Committee on Cancer (AJCC) 7th edition (2010) and Japanese Research Society for Gastric Cancer (JRSGC), 3rd edition (2011), our study shows a distribution of cancer stage as follows: IA, IB, IIA, IIB, IIIA, IIIB and IIIC, with proportions of $4.3 \%$, $11.4 \%, 32.9 \%, 30 \%, 7.1 \%, 11.4 \%$ and $2.9 \%$, respectively. The authors also comment that the majority of gastric cancers were observed at stages II and III.

\section{Intraoperative complications}

Our study had 01 (1.4\%) case of liver damage during liver transfusion, causing left hepatic parenchyma bleeding, 01 (1.4\%) case of splenic injury when releasing the omentum, causing bleeding, and $01(1.4 \%)$ case of a small intestine muscle tear when performing suturing for gastrointestinal anastomosis; all were treated with haemostasis by electrocautery and inner muscular suturing during laparoscopic surgery. No deaths occurred intra-operatively. The rates of intraoperative complications of authors worldwide have been reported to range from $0.9-7.4 \%(3,4,17,18)$.

\section{Postoperative complications}

$01(1.4 \%)$ case developed residual abscess under the left liver after 5 days of surgery. The patient manifested fever, and fluid agglomerate $3 \times 4 \times 2 \mathrm{~cm}$ in size was detected below the left liver via abdominal ultrasound. The patient then received pus aspiration of $100 \mathrm{ml}$ in accordance with ultrasound guidance. After receiving aspiration with stable internal medicine, the patient was discharged on the 13th day after surgery. Furthermore, 01 (1.4\%) case of pneumonia occurred after surgery on $4^{\text {th }}$ day; the patient underwent conservative treatment in internal medicine and was discharged on the $10^{\text {th }}$ day. No deaths occurred postoperatively. The rates of complications reported by Western authors fluctuate between $21-26 \%(7,16)$. With regard to postoperative pain, we found that $60 \%$ of patients experienced minor pain, 38.6\% moderate pain, and no cases experienced major pain. After surery, conventional painkillers were used routinely in our study. Some cases used painkillers for epidural pain after surgery; however, we observed that most patients had low and moderate pain. If the patients suffered much pain, morphine painkillers were used as supplemental medicine. We noted that almost all patients after laparoscopic surgery felt comfortable and desired to use fewer painkillers and have faster movement recovery.

The mean operating time was $206.8 \pm 27.2$ minutes. The first cases had longer surgical time, approximately 300 minutes; after that, the surgical time became shorter and shorter in later cases. This result is also consistent with previous studies in the literature $(17,19)$. The mean time to first flatus was recorded as $55.3 \pm 20.9$ hours, which was lower than the result of the study conducted by Noshiro et al (67.2 hours) (20). The mean duration of hospital stay in our study was $8.8 \pm 2.4$ days, with the lowest and highest times of 6 days and 15 days, respectively. Similarly, Chen K's study and Lee SE's study followed patients who received laparoscopic distal gastrectomy and suggested postoperative hospitalization times of $9.8 \pm 3.7$ days and $8.0 \pm 3.3$ days, respectively (21), (22). Conversely, Haverkamp et al, in a meta-analysis of 698 cases, found an average length of postoperative hospital stay of approximately four days of difference in favour of laparoscopy compared to open gastrectomy, at 14.1 and 18.1 days, respectively $(p<0.001$ ). The mean times for gastric catheter withdrawal and intra-abdominal drain removal were $50.1 \pm 34.6$ hours and $4.05 \pm 1.3$ days, respectively. Nasogastric intubation is an issue that makes patients very uncomfortable and affects their quality of life. Through the study, we realized that no anastomotic bleeding or gastrointestinal leakage events occurred in 70 patients when they received gastrointestinal tract suturing by stapler; therefore, it is not necessary to have nasogastric intubation 24 hours after surgery. Our study presented 8 cases without nasogastric intubation and 22 with 24-hour postoperative nasogastric intubation, of which there were no cases of anastomotic complications. The overall survival rates after 1 year and 3 years were $97.7 \%$ and $83.8 \%$, respectively. The mean overall survival was estimated as $43.8 \pm 1.5$ months, ranging from $40.9-46.7$ months. Chen et al conducted laparoscopic distal gastrectomy on 240 gastric cancer patients and reported a 1-year overall survival rate of $91.5 \%$ (21). The overall survival reported by Vietnamese authors ranged from 40.243.8 months. 


\section{CONCLUSION}

A study of 70 patients undergoing laparoscopic surgery with D2 lymphadenectomy with left-site surgeon and resection - closure of the duodenal stump in final phase after making esophageal anastomosis in gastric cancer treatment found that the mean age was 59.9 years old, and the male/female ratio was $4 / 1$. Gastric cancer was mainly staged at IA, IB, IIA, IIB, IIIA, and IIIB, the average number of retrieved lymph nodes was 21.5 , and the mean number of metastatic lymph nodes was 2.6 nodes. The lymph node stations with high incidence rates of metastases were Nos. 1, 2, 3, 4 and 7. The mean operation time was 206.8 minutes, the rates of intraoperative and postoperative complications were low, there were no deaths during and after surgery, and the average hospital stay was 8.8 days. Laparoscopic total gastrectomy with D2 lymph node dissection with left-site surgeon and resection - closure of the duodenal stump in final phase after making esophageal anastomosis in gastric cancer treatment is a safe, effective and highly aesthetic method with decreased pain for the patient, faster movement recovery and shortened hospital stay. The mean overall survival was 43.8 months.

\section{Ethical approval}

The clinical trial was approved by the hospital's ethics committee and was conducted in accordance with the Helsinki Declaration.

\section{Conflict of interest}

\section{All author declare that they have no conflict of interest.}

\section{REFFERENCES}

1. Bray F, Ferlay J, Soerjomataram I, Siegel RL, Torre LA, Jemal A. Global cancer statistics 2018: GLOBOCAN estimates of incidence and mortality worldwide for 36 cancers in 185 countries. CA Cancer J Clin. 2018:68(6):394-424. Epub 2018 Sep 12.

2. Azagra JS, Groergen M, Gilbart E, Alonso. J, Centerik M (2001) Laparoscopy assisted total gastrectomy with extended D2 lymphadenectomy for cancer: Technical aspect". Le Jour Coelio- Chir, 40: 79- 83.

3. Ye L-y, Liu D-r, Li C, Li X-w, Huang L-n, Ye S, et al. Systematic review of laparoscopy - assisted versus open gastrectomy for advanced gastric cancer. J Zhejiang Univ Sci B. 2013;14(6):468-78.

4. Chen K, Xu X-W, Zhang R-C, Pan Y, Wu D, Mou Y-P. Systematic review and meta - analysis of laparoscopy assisted and open total gastrectomy for gastric cancer. World J Gastroenterol. 2013;19(32) 5365-76.

5. Chen K, Pan Y, Cai J-Q, Xu X-W, Wu D, Mou Y-P. Totally laparoscopic gastrectomy for gastric cancer: A systematic review and metaanalysis of outcomes compared with open surgery. World $\mathrm{J}$ Gastroenterol. 2014; 20(42):15867-78.

6. Matsuhashi N, Osada S, Yamaguchi K, Saito S, Okumura N, Tanaka Y, et al. Oncologic outcomes of laparoscopic gastrectomy: a singlecenter safety and feasibility study. Surg Endosc. 2013;27(6):1973-9.

7. Huscher CGS, Mingoli A, Sgarzini G, Sansonetti A, Di Paola M, Recher A , et al. Laparoscopic versus open subtotal gastrectomy for distal gastric cancer: five-year results of a randomized prospective trial. Ann Surg. 2005;241(2):232-7.

8. Kim H-H, Han S-U, Kim M-C, Hyung WJ, Kim W, Lee H-J, et al. Long-term results of laparoscopic gastrectomy for gastric cancer: a large-scale case-control and case-matched korean multicenter study. J Clin Oncol. 2014;32(7):627-33.

9. Tsujimoto H, Uyama I, Yaguchi Y, Kumano I, Takahata R, Matsumoto $\mathrm{Y}$, et al. Outcome of Overlap anastomosis using a linear ar stapler after laparoscopis total and proximal gastrectomy. Langenbecks Arch Surg. 2012;397(5):833-40.

10. Zhao S, Zheng K, Zheng J-C, Hou T-T, Wang Z-N, Xu H-M, et al. Comparison of totally laparoscopic total gastrectomy and laparoscopic-assisted total gastrectomy : A systematic review and meta-analysis. Int J Surg. 2019;68:1-10.

11. Liao T, Deng L, Yao X, Ouyang M. Comparison of the safety and efficacy between linear stapler and circular stapler in totally laparoscopic total gastrectomy, Protocol for a systematic review and meta-analysis. BMJ Open. 2019;9(5):e028216.

12. Kang S-H, Cho Y-S, Min S-H, Park YS, Ahn S-H, Park DJ, et al. Intracorporeal esophagojejunostomy uing a circular or a linear stapler in totally laparoscopic total gastrectomy, a propensitymatched analysis. J Gastric Cancer. 2019;19(2):193-201.

13. Japanese Gastric Cancer Association. Japanese classification of gastric carcinoma - 3rd english edition. Gastric Cancer. 2011; 14(2):101-12.

14. Japanese Gastric Cancer Association. Japanese gastric cancer treatment guidelines 2014 (ver. 4). Gastric Cancer. 2017;20(1):1-19. Epub 2016 Jun 24

15. Japanese Gastric Cancer Association. Japanese gastric cancer treatment guidelines 2018 (5th edition). Gastric Cancer. 2020 Feb 14. Online ahead of print.

16. Strong VE, Devaud N, Allen PJ, Gonen M, Brennan MF, Coit D. Laparoscopic versus open subtotal gastrectomy for adenocarcinoma: a case - control study. Ann Surg Oncol. 2009;16(6):1507-13

17. Kitano S, Shiraishi N, Uyama I, Sugihara K, Tanigawa N, Japanese Laparoscopic Surgery Study Group. A multicenter study on oncologic outcome of laparoscopic gastrectomy for early cancer in japan. Ann Surg. 2007;245(1):68-72.

18. Han G, Park JY, Kim YJ. Comparison of short-term postoperative outcomes in totally laparoscopic distal gastrectomy versus laparoscopy-assisted distal gastrectomy. J Gastric Cancer. 2014; 14(2):105-10.

19. Chen QY, Huang CM, Lin JX, Zheng CH, Li P, Xie JW, et al. Laparoscopy-assisted versus open D2 radical gastrectomy for advanced gastric cancer without serosal invasion: a case control study. World J Surg Oncol. 2012;10:248.

20. Noshiro $H$, Nagai $E$, Shimizu S, Uchiyama A, Tanaka M. Laproscopicallly assisted distal gastrectomy whith standard radical lymph node dissection for gastric cancer. Surg Endosc. 2005; 19(12):1592-6.

21. Chen K, Mou YP, Xu XW, Cai JQ, Wu D, Pan Y, et al. Short-term surgical and long-term survival outcomes after laparoscopic distal gastrectomy with D2 lymphadenectomy for gastric cancer. BMC Gastroenterol. 2014;14:41.

22. Lee SE, Kim YW, Lee JH, Ryu KW, Cho SJ, Lee JY, et al. Developing an institutional protocol guideline for laparoscopy-assisted distal gastrectomy. Ann Surg Oncol. 2009;16(8):2231-6.

23. Haverkamp L, Weijs TJ, van der Sluis PC, van der Tweel I, Ruurda $J P$, van Hillegersberg R. Laparoscopic total gastrectomy versus open total gastrectomy for cancer: a systematic review and metaanalysis. Surg Endosc. 2013;27(5):1509-20 